\title{
Oblique cord (chorda obliqua) of the forearm and muscle-associated fibrous tissues at and around the elbow joint: a study of human foetal specimens
}

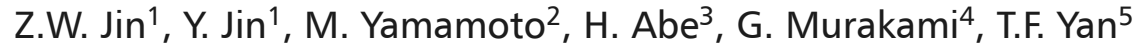 \\ ${ }^{1}$ Department of Anatomy, Histology and Embryology, Yanbian University Medical College, China \\ 2Department of Anatomy, Tokyo Dental College, Japan \\ ${ }^{3}$ Department of Anatomy, Akita University Graduate School of Medicine, Japan \\ ${ }^{4}$ Division of Internal Medicine, Iwamizawa Kojin-kai Hospital, Japan \\ ${ }^{5}$ Division of Gynaecology and Obstetrics, Baishan Central Hospital, China
}

[Received: 11 January 2016; Accepted: 13 February 2016]

In adults, the oblique cord or chorda obliqua separates the origins of the flexor pollicis longus (FPL) and flexor digitorum profundus (FDP) muscles from the supinator muscle and elbow joint. This study examined the topographic anatomy of the oblique cord and related muscles in foetuses. Semiserial sections of five mid-term foetuses of gestational age (GA) 14-16 weeks and 12 late-stage foetuses of GA 28-30 weeks were histologically examined and three forearms at GA 30 weeks were macroscopically evaluated. Late-stage foetuses showed a fascial structure between the supinator and FDP muscles. The latter extended proximally to the elbow joint and the muscle origin thickened the distal, ulnar part of the capsule. The FPL origin also extended proximally but did not reach the joint capsule. These morphologies were consistent with macroscopic examinations. The brachialis muscle was widely inserted into the proximal, anterior part of the capsule. In addition, the medial collateral ligament was not covered by the pronator-flexor muscles but by the triceps brachii muscle. The oblique cord apparently did not form prenatally. After birth, the proximal parts of the FDP and FPL muscles were likely replaced by collagenous tissues, providing a specific type of intermuscular septum i.e., the oblique cord. This type of muscle-ligament transition was observed in the annular ligament of the radius. The foetal elbow joint was characterised by strong support by the FDP, brachialis and triceps brachii muscles. Therefore, the foetal elbow is not a miniature version of the adult elbow. (Folia Morphol 2016; 75, 4: 493-502)

Key words: oblique cord, elbow joint capsule, flexor digitorum profundus muscle, brachialis muscle, collateral ligament, human foetuses

\section{INTRODUCTION}

The oblique cord or chorda obliqua of the forearm is a flat fascial band along the distal border of the deep head of the spinator muscle, extending from the lateral side of the ulnar tuberosity to the radius slightly distal to its tuberosity [35].
Thus, in adults, the oblique cord is attached to the flexor pollicis longus (FLP) and flexor digitorum profundus (FDP) muscles from the ulnar-distal to the radial-proximal side [11, 14]. Figure 1 shows the topographical anatomy around the oblique cord in adults: the cord separates the FPL and FDP muscles

Address for correspondence: Y. Jin, MD, PhD, Department of Anatomy, Histology and Embryology, Yanbian University Medical College, No. 977 Gongyuan Road, Yanji, Jilin, 133002, China, e-mail: yujinyb@sina.com 
from the supinator muscle as well as from the elbow joint capsule. Conversely, the origins of the FDP and FPL muscles do not reach the joint capsule in adults.

The interosseous membrane of the forearm is most taut at the neutral position of the hand [5, $18,28]$ and it plays a role in load transfer from the radius to the ulna during axial loading activities [15, $25,27]$. In contrast, because the fibre direction is at a right angle to the fibres in the interosseous membrane, the oblique cord is most taut during supination and is unlikely to play a role in axial load transfer from the radius to the ulna. Therefore, its functional significance is unclear [35]. A comparative anatomical study suggested that the oblique cord is a remnant of the elbow stabiliser in quadrupedal primates [24]. As the oblique cord is sandwiched between the supinator and the FDP and FPL muscles (Fig. 1), it is likely to develop as an intermuscular septum. The first aim of this study was therefore to assess the foetal development of the cord, regarding the mechanical stress required to induce formation of the distinct intermuscular septum between the supinator and flexor muscles.

The adult elbow joint is surrounded by many muscles. With strong assistance from the flexorpronator muscle complex, originating from the epicondylus medialis of the humerus, the medial collateral ligament is thought to stabilise the elbow joint against valgus stress [7, 22]. In contrast, the lateral collateral ligament complex of the elbow prevents posterolateral rotator instability, strongly assisted by the lateral ulnar collateral ligament extending between the anconeus and extensor carpi ulnaris muscles $[12,16,21]$. Therefore, intermuscular septa, possibly including the oblique cord, are likely to provide a muscle-ligament complex for joint stabilisation. However, despite its close relationship to the joint capsule, the cord is usually ignored. Therefore, the second aim of this study was to compare the oblique cord with the other muscle-associated fibrous tissues located at and around the elbow joint.

\section{MATERIALS AND METHODS}

The study was performed in accordance with the provisions of the Declaration of Helsinki 1995 (as revised in 2013). Semiserial paraffin sections of the unilateral elbow were obtained from five midterm human foetuses of gestational age (GA) 14-16 weeks (crown-rump length [CRL] 100-125 mm) and from 12 late-stage foetuses of GA 28-30 weeks (CRL

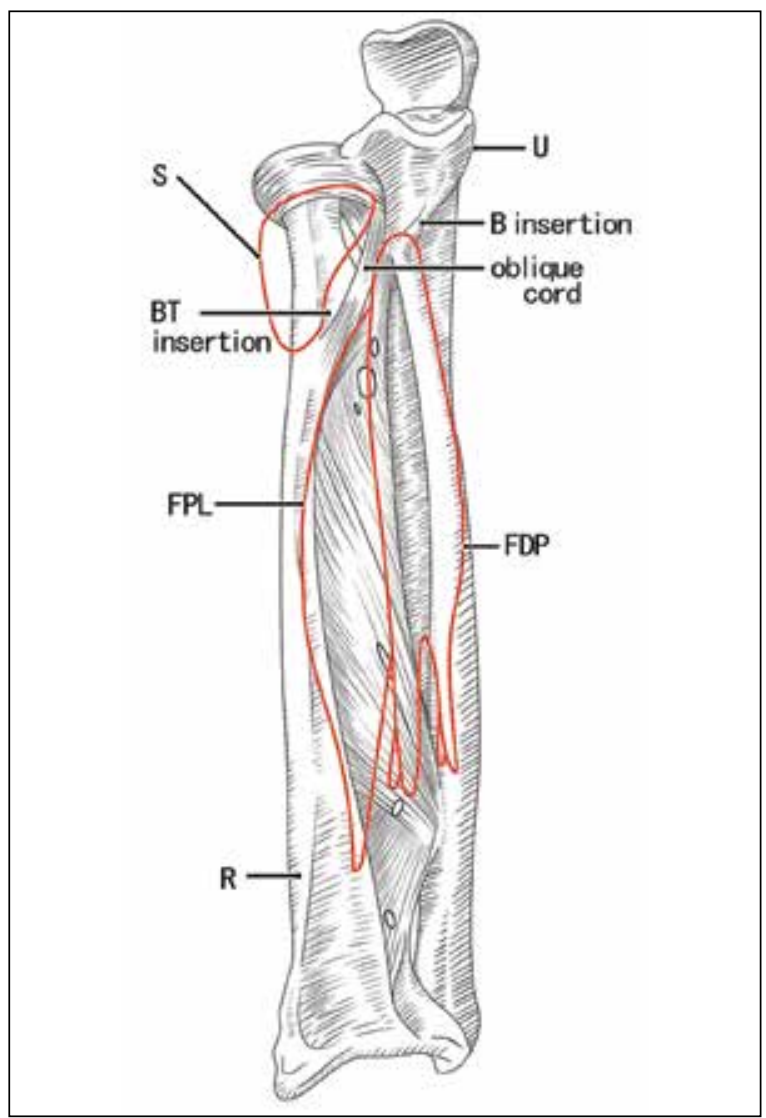

Figure 1. A schematic representation showing topographical relation between the oblique cord and the muscle origins and insertions. The oblique cord or chorda obliqua is located between the supinator muscle and the flexor pollicis longus muscle (FPL) and flexor digitorum profundus muscle (FDP) in adults. The insertions of the biceps tendon (BT) and the brachialis muscle $(\mathrm{B})$ are in the radial and ulnar side of the cord, respectively; $\mathrm{R}$ - radius; $\mathrm{S}$ supinator muscle; U — ulna.

230-255 mm). All of the sectional planes were longitudinal along the long axis of the forearm; however, precise sagittal and frontal planes were not obtained because of pronation at various degrees.

The 12 late stage foetuses were parts of a collection in Department of Anatomy, Akita University, Akita, Japan. They were donated by their families to the Department during 1975-1985 and preserved in $10 \% \mathrm{w} / \mathrm{w}$ neutral formalin solution for more than 30 years. The available data was limited to the date of donation and the gestational weeks, but we did not find a document saying the family name, the name of obstetricians or hospital and the reason of abortion. After dividing the lower extremities from the body, the specimens were decalcified by incubating at room temperature using Plank-Rychlo solution $\left(\mathrm{AlCl}_{2} / 6 \mathrm{H}_{2} \mathrm{O}, 7.0 \mathrm{w} / \mathrm{v} \% ; \mathrm{HCl}\right.$, 
3.6; $\mathrm{HCOOH}, 4.6)$ for 1 week. The sectional planes were longitudinal (almost sagittal) and stained with haematoxylin and eosin (HE). Due to highly acidic decalcification, the Akita specimens were not available for immunohistochemistry. In addition, three upper extremities from three of the Akita foetuses of GA 30 weeks, preserved in 10\% formalin, were used for macroscopic observations. The dissection started from the flexor side of the forearm to reach the interosseous membrane. The use for research was approved by the University Ethics Committee in Akita (No. 1378).

The 5 mid-term specimens ( 1 at GA 14 weeks; 2 at GA 15 weeks; and 2 at GA 16 weeks) were donated by their families to the Department of Anatomy, Yanbian University Medical College, Yanji, China, and their use for research was approved by the Yanbian University Ethics Committee (No. BS-13-35). These foetuses were obtained by induced abortion, after which the mother was orally informed by an obstetrician at the college teaching hospital of the possibility of donating the foetus for research; no attempt was made to actively encourage the donation. After the mother agreed, the foetus was assigned a specimen number and stored in $10 \% \mathrm{w} / \mathrm{w}$ neutral formalin solution for more than 1 month. Because of specimen number randomisation, there was no possibility of contacting the family at a later date. After dividing the body into parts, pelvic samples were decalcified by incubation at $4^{\circ} \mathrm{C}$ in $0.5-\mathrm{mol} / \mathrm{L}$ EDTA ( $\mathrm{pH} 7.5$ ) solution (Decalcifying Solution B; Wako, Tokyo, Japan) for 3-5 days, depending on the size of the sample. Most sections were stained with $\mathrm{HE}$, while some were reserved for immunohistochemistry.

For immunohistochemistry, antigen was retrieved from tissue sections by microwave treatment ( $500 \mathrm{~W}, 15 \mathrm{~min}, \mathrm{pH}$ 6). Sections were incubated with primary antibodies, including 1) mouse monoclonal anti-human desmin (dilution, 1:50; Dako N1526, Glostrup, Denmark); 2) mouse monoclonal antihuman vimentin (Dako M7020, 1:10); and 3) mouse monoclonal anti-human CD68 KP1 (Dako M0814, 1:100). After washing, the sections were incubated with secondary antibody (Dako Chem Mate Envison Kit), labelled with horseradish peroxidase (HRP), and antigen-antibody reactions were detected by the HRP-catalysed reaction with diaminobenzidine. All samples were counterstained with haematoxylin. Each experiment included a negative control, con- sisting of tissue specimens without primary antibody. Sections were observed and photographed with a Nikon Eclipse 80. However, photographs at ultralow magnification (less than $\times 1$ at the objective lens) were taken using a high grade flat scanner (Epson GTX970) with translucent illumination.

\section{RESULTS}

\section{Histological observations}

Figures 2-4 show sections from the mid-term foetuses, while Figures 5-7 show those from the late stage foetuses. Figure 4 shows the immunohistochemistry results of a border area between the supinator muscle and the FDP or FPL muscle. Figure 7 shows higher magnifications of some panels in Figures 5 and 6 . In Figures 2, 3, 5 and 6, the longitudinal sections are arranged from the radial side to the ulnar side. Muscles were identified in these longitudinal sections relative to 1) tendons toward the hand and wrist and 2) the median, ulnar and radial nerve trunks. Conversely, at and near the elbow, a muscle without long tendon(s) corresponded to the supinator, pronator teres or anconeus muscle. The anconeus muscle was continuous with the triceps brachii muscle (Figs. 5B, 6B). The median nerve passed through the pronator teres muscle and ran between the deep and superficial flexors (Figs. 2D, E; $3 \mathrm{C}-\mathrm{E} ; 5 \mathrm{E}, \mathrm{F} ; 6 \mathrm{E})$. The radial nerve ran between the brachialis and extensor muscles and passed through the supinator muscle (Figs. 2A, B; 3A; 5AB;6B). The ulnar nerve ran between the flexor carpi ulnaris and flexor digitorum superficialis muscles (Figs. 2E, $F$; $3 E, F ; 5 F, G ; 6 F, G)$. Muscle attachment to the joint capsule could easily be determined in the longitudinal sections.

Examination of the area in which the oblique cord was expected, i.e., the border between the supinator and FDP muscles, showed that this area in both mid-term and late stage specimens was filled with a thin fascia or loose tissue containing vessels (Figs. 2A-C; 3B, C; 4A; 5C, D; 6C). This thin fascia or loose tissue, positive for vimentin but negative for desmin, did not contain C68-positive macrophages (Fig. 4B-D). In contrast to the FDP, the FPL did not usually face the supinator, being observed in only one mid-term (Fig. 2B) and 6 late stage foetuses. Rather, the FPL usually faced the insertion of the pronator teres muscle into the distal side of the supinator muscle. Near the insertion, the biceps 

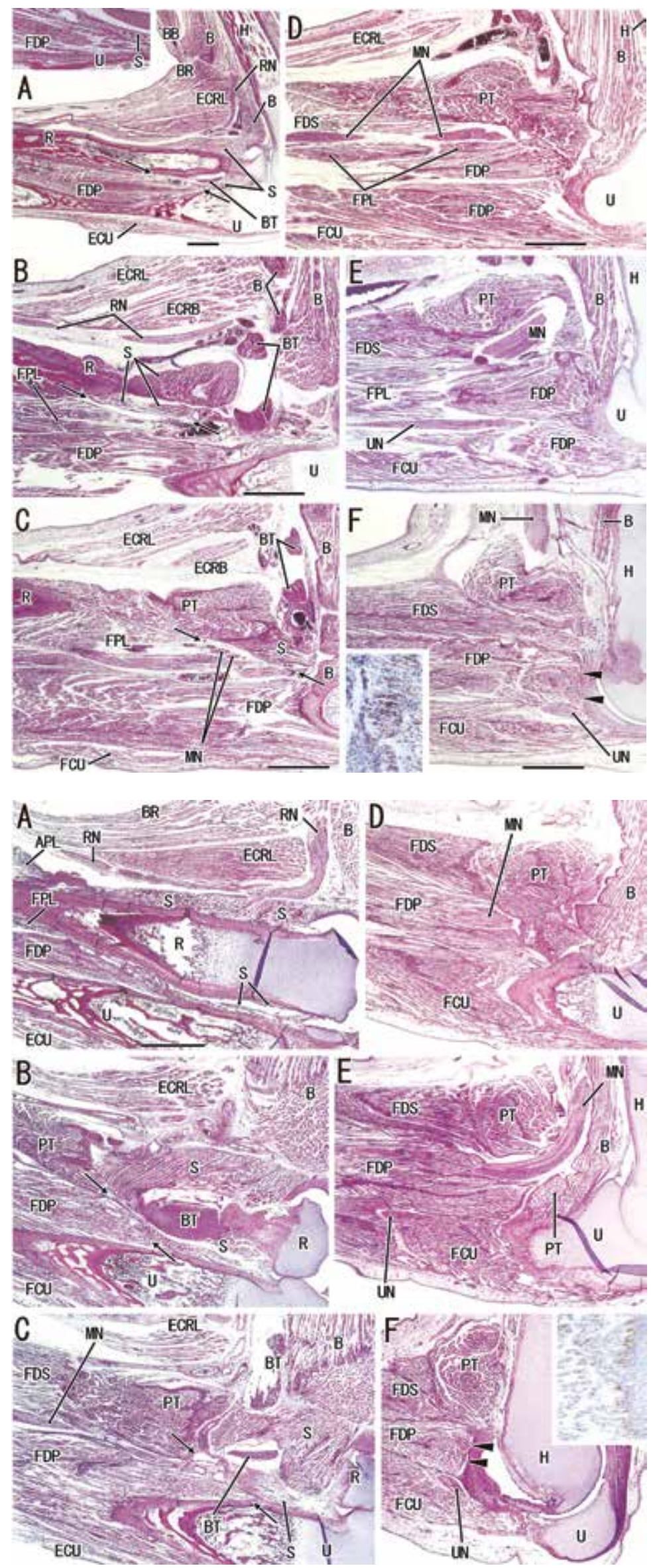

Figure 2. Elbow and forearm at 15 weeks. Panel A (panel F) displays the most lateral (medial) site in the figure. Intervals between panels are $0.6 \mathrm{~mm}(A-B$, $B-C, C-D, D-E)$ and $0.9 \mathrm{~mm}(E-F)$. Arrows in panels A-C indicate an area in which the oblique cord is expected to develop. Insert in panel $\mathbf{A}$ displays the higher magnification view of the area. The radial nerve (RN) enters the forearm in panels $\mathbf{A}$ and $\mathbf{B}$. The median nerve (MN) runs between the flexor digitorum profundus and superficialis muscles (FDP, FDS) in panels $\mathbf{D}$ and $\mathbf{E}$. The ulnar nerve passes through the flexor carpi ulnaris muscle (FCU) in panels $\mathbf{E}$ and $\mathbf{F}$. Arrowhead in panel $\mathbf{F}$ indicates the proximal origin of the flexor digitorum profundus (FDP) muscle from the joint capsule. An insert in panel $\mathbf{F}$ exhibits immunohistochemistry of desmin at the FDP origin (a near section of panel $\mathbf{F}$ ). All panels are prepared at the same magnification (scale bar in panel $A$ : $1 \mathrm{~mm}) ; \mathrm{B}$ - brachialis muscle; $\mathrm{BB}$ - biceps brachii muscle; $\mathrm{BR}$ - brachioradialis muscle; $\mathrm{BT}$ - biceps tendon; ECRL - extensor carpi radialis longus muscles; ECRB - extensor carpi radialis brevis muscles; ECU — extensor carpi ulnaris muscle; FPL — flexor pollicis longus muscle; $\mathrm{H}$ - humerus; $\mathrm{PT}$ - pronator teres muscle; $\mathrm{R}$ - radius; $\mathrm{S}$ - supinator muscle; $\mathrm{U}$ - ulna; UN — ulnar nerve.

Figure 3. Elbow and forearm at 16 weeks. Panel A (panel F) displays the most lateral (medial) site in the figure. Intervals between panels are $0.7 \mathrm{~mm}(\mathrm{~A}-\mathrm{B})$, $0.3 \mathrm{~mm}(\mathrm{~B}-\mathrm{C}), 0.9 \mathrm{~mm}(\mathrm{C}-\mathrm{D}), 0.7 \mathrm{~mm}(\mathrm{D}-\mathrm{E})$ and $0.4 \mathrm{~mm}(\mathrm{E}-\mathrm{F})$. Arrows in panels $\mathbf{B}$ and $\mathbf{C}$ indicate an area in which the oblique cord is expected to develop. The radial nerve (RN) enters the forearm in panel A. The median nerve (MN) enters the forearm in panel $\mathbf{E}$ and runs between the pronator teres muscle (PR) and flexor digitorum profundus (FDP) muscle in panel C. The ulnar nerve passes between the flexor carpi ulnaris muscle (FCU) and FDP muscle in panels $\mathbf{E}$ and $\mathbf{F}$. Arrowhead in panel $\mathbf{F}$ indicates the proximal origin of the FDP muscle from the joint capsule. An insert in panel $\mathbf{F}$ exhibits immunohistochemistry of desmin at the FDP origin (a near section of panel $\mathbf{F}$ ). All scale bars: $1 \mathrm{~mm}$. Other abbreviations - see the common abbreviation for figures; APL — abductor pollicis longus muscle; $\mathrm{B}$ - brachialis muscle; $\mathrm{BR}$ - brachioradialis muscle; $\mathrm{BT}$ - biceps tendon; ECRL - extensor carpi radialis longus muscles; ECU - extensor carpi ulnaris muscle; FDS — flexor digitorum superficialis muscle; FPL — flexor pollicis longus muscle; $\mathrm{H}$ - humerus; $\mathrm{PT}$ - pronator teres muscle; $\mathrm{R}$ - radius; $\mathrm{S}$ - supinator muscle; $\mathrm{U}-$ ulna; UN - ulnar nerve. 
Figure 4. Immunohistochemistry of an area in which the oblique cord is expected to develop. A specimen at 15 weeks but different from that shown in Figure 1. All panels display near sections; panel A: HE staining; panel B: immunohistochemistry of vimentin; panel $\mathbf{C}$ : immunohistochemistry of desmin; panel $\mathbf{D}$ : immunohistochemistry of CD68. Arrows in panels $A-D$ indicate a border area between the supinator muscle (S) and the flexor digitorum profundus (FDP) muscle: therein, the oblique cord is expected to develop. This area is filled with a loose fibrous tissue (vimentin+, desmin-). CD68-positve macrophages are absent in the area. All scale bars, $1 \mathrm{~mm}$. B - brachialis muscle; BR - brachioradialis muscle; $\mathrm{ECRL}$ - extensor carpi radialis longus muscles; ECRB extensor carpi radialis brevis muscles; FCU — flexor carpi ulnaris muscle; $\mathrm{FPL}$ - flexor pollicis longus muscle; $\mathrm{R}$ radius; $\mathrm{RN}$ — radial nerve; $\mathrm{U}$ — ulna.

Figure 5. Elbow and forearm at 28 weeks. Panel A (panel G) displays the most lateral (medial) site in the figure. Intervals between panels are $2.0 \mathrm{~mm}(\mathrm{~A}-\mathrm{B}), 0.6 \mathrm{~mm}(\mathrm{~B}-\mathrm{C}, \mathrm{C}-\mathrm{D})$, $1.0 \mathrm{~mm}(\mathrm{D}-\mathrm{E}), 2.4 \mathrm{~mm}(\mathrm{E}-\mathrm{F})$ and $0.5 \mathrm{~mm}(\mathrm{~F}-\mathrm{G})$. Arrows in panels $\mathbf{C}$ and $\mathbf{D}$ indicate an area in which the oblique cord is expected to develop. The radial nerve (RN) passes through the supinator muscle $(S)$ in panel $\mathbf{B}$ and runs between the supinator muscle $(S)$ and the extensor carpi radialis muscles (ECRL, ECRL) in panels $\mathbf{C}$ and $\mathbf{D}$. The median nerve (MN) passes through the pronator teres muscle (PT) in panel $\mathbf{E}$. The ulnar nerve passes between the flexor carpi ulnaris and flexor digitorum profundus muscles (FCU, FDP) in panels $\mathbf{F}$ and G. The medial collateral ligament is covered by the triceps brachii muscle (TB) in panels $\mathbf{F}$ and $\mathbf{G}$ : it will be shown at the higher magnification in Figure 7E. Arrowhead in panel $\mathbf{F}$ indicates the proximal origin of the flexor digitorum profundus muscle from the joint capsule. All panels are prepared the same magnification (scale bar in panel E: $5 \mathrm{~mm}$ ); $A$ - anconeus muscle; $\mathrm{B}$ - brachialis muscle; $\mathrm{BB}$ - biceps brachii muscle; $\mathrm{BR}$ - brachioradialis muscle; $\mathrm{BT}$ - biceps tendon; ECRB - extensor carpi radialis brevis muscles; ECU extensor carpi ulnaris muscle; EMH - epicondylus medialis of the humerus; $\mathrm{H}$ - humerus; $\mathrm{MCL}$ - medial collateral ligament; $\mathrm{R}$ — radius; $\mathrm{U}$ — ulna; UN — ulnar nerve.
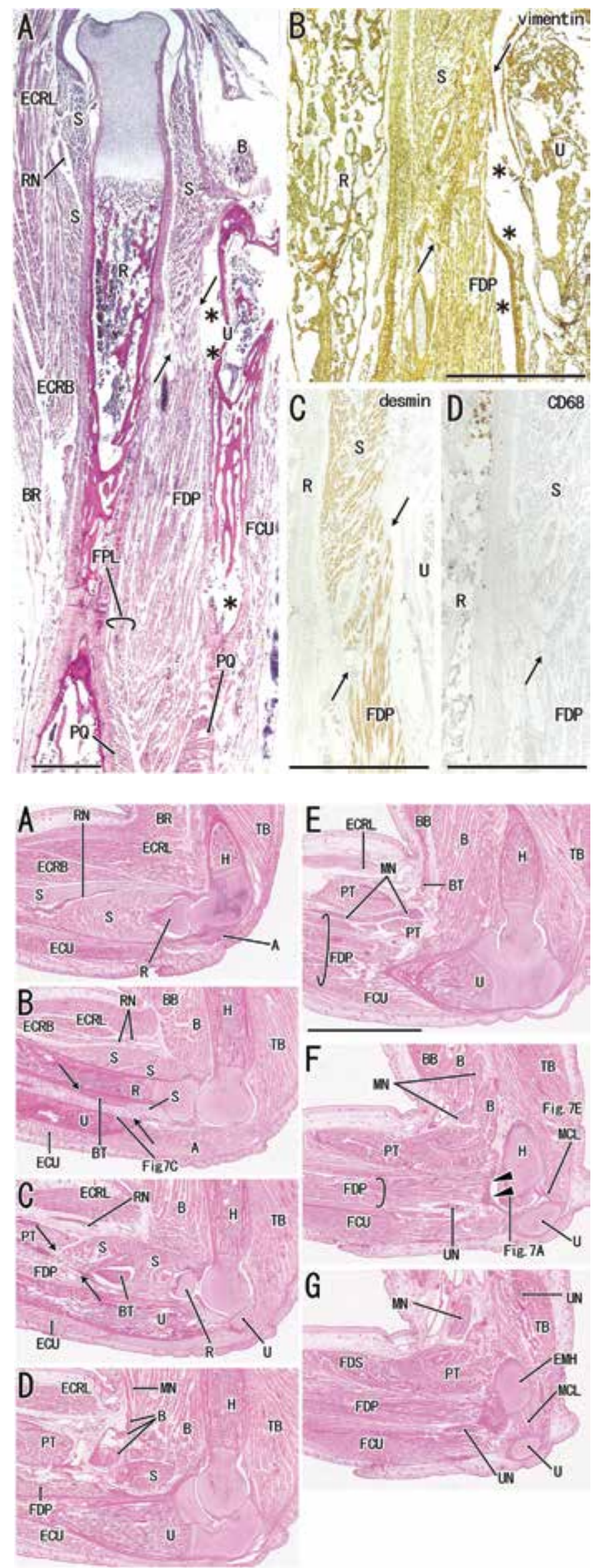


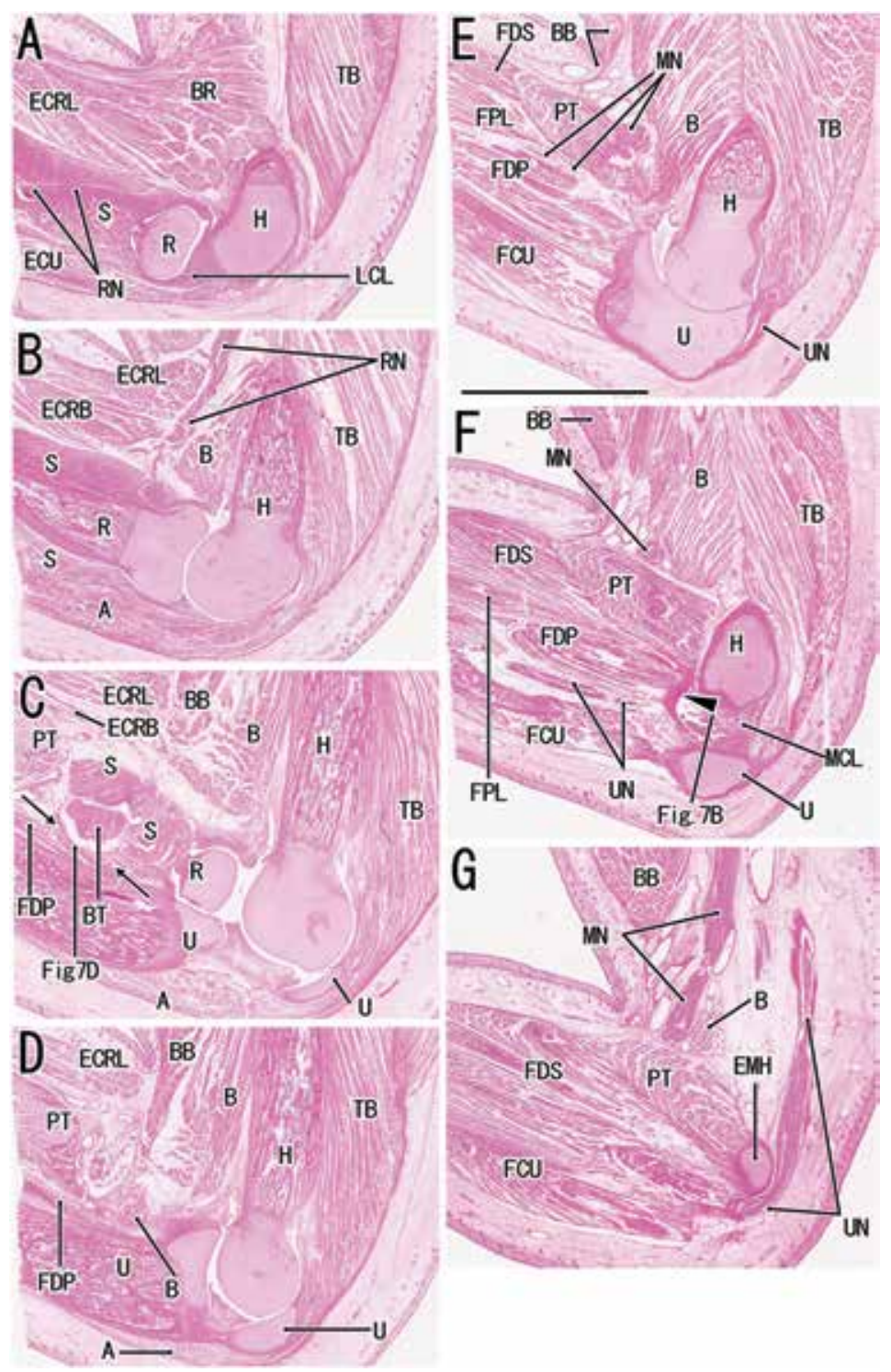

Figure 6. Elbow and forearm at 30 weeks. Panel $\mathbf{A}$ (panel G) displays the most lateral (medial) site in the figure. Intervals between panels are $1.6 \mathrm{~mm}(A-B), 1.5 \mathrm{~mm}(B-C), 0.9 \mathrm{~mm}(C-D), 2.7 \mathrm{~mm}(D-E)$ and $1.1 \mathrm{~mm}(E-F, F-G)$. Arrows in panel $C$ indicate an area in which the oblique cord is expected to develop. The radial nerve (RN) enters the forearm in panel $\mathbf{B}$. The median nerve (MN) enters the forearm in panel $\mathbf{F}$ and runs between the pronator teres and flexor digitorum profundus muscles (PT, FDP) in panel $\mathbf{E}$. The ulnar nerve curves along the epicondylus medialis of the humerus (EMH) in panel $\mathbf{G}$ and runs between the FCU and FDP muscles in panel $\mathbf{F}$. The medial collateral ligament is covered by the triceps brachii muscle (TB) in panel $\mathbf{F}$ : it will be shown at the higher magnification in Figure 7E. Arrowhead in panel $\mathbf{F}$ indicates the proximal origin of the flexor digitorum profundus muscle from the joint capsule. All panels are prepared at the same magnification (scale bar in panel $\mathrm{E}: 5 \mathrm{~mm}$ ); $\mathrm{A}$ — anconeus muscle; $\mathrm{B}$ — brachialis muscle; $\mathrm{BB}$ - biceps brachii muscle; $\mathrm{BR}$ — brachioradialis muscle; $\mathrm{BT}$ — biceps tendon; ECRL — extensor carpi radialis longus muscles; ECRB - extensor carpi radialis brevis muscles; ECU — extensor carpi ulnaris muscle; FDS — flexor digitorum superficialis muscle; $\mathrm{FPL}$ - flexor pollicis longus muscle; $\mathrm{H}$ - humerus; $\mathrm{LCL}$ - lateral collateral ligament; $\mathrm{R}$ radius; $\mathrm{S}$ - supinator muscle; $\mathrm{U}$ - ulna; UN — ulnar nerve.

tendon was located close to the border area, but its sheath was very thin (Figs. 2B; 3C; 6C) or indistinct (Fig. 5C, D). Notably, the FDP extended proximally to attach to the humeroulnar joint capsule (Fig. 2F; $3 F ; 5 F ; 6 F$ ). At the attachment site (insert of Figs. $2 \mathrm{~F} ; 3 \mathrm{~F}$ ), the muscle fibres of the FDP contained the so-called desmin-spots, suggesting muscle origin or insertion according to our previous study [3]. HE staining of the late stage foetuses showed the FDP origin from the capsule (Fig. 7A, B).

The FDP origin thickened the distal, ulnar part of the capsule (Fig. 7A, B). In contrast, wide insertion of the brachialis muscle was evident at the proximal, anterior part of the capsule (Figs. 5F; 6F). Moreover, 


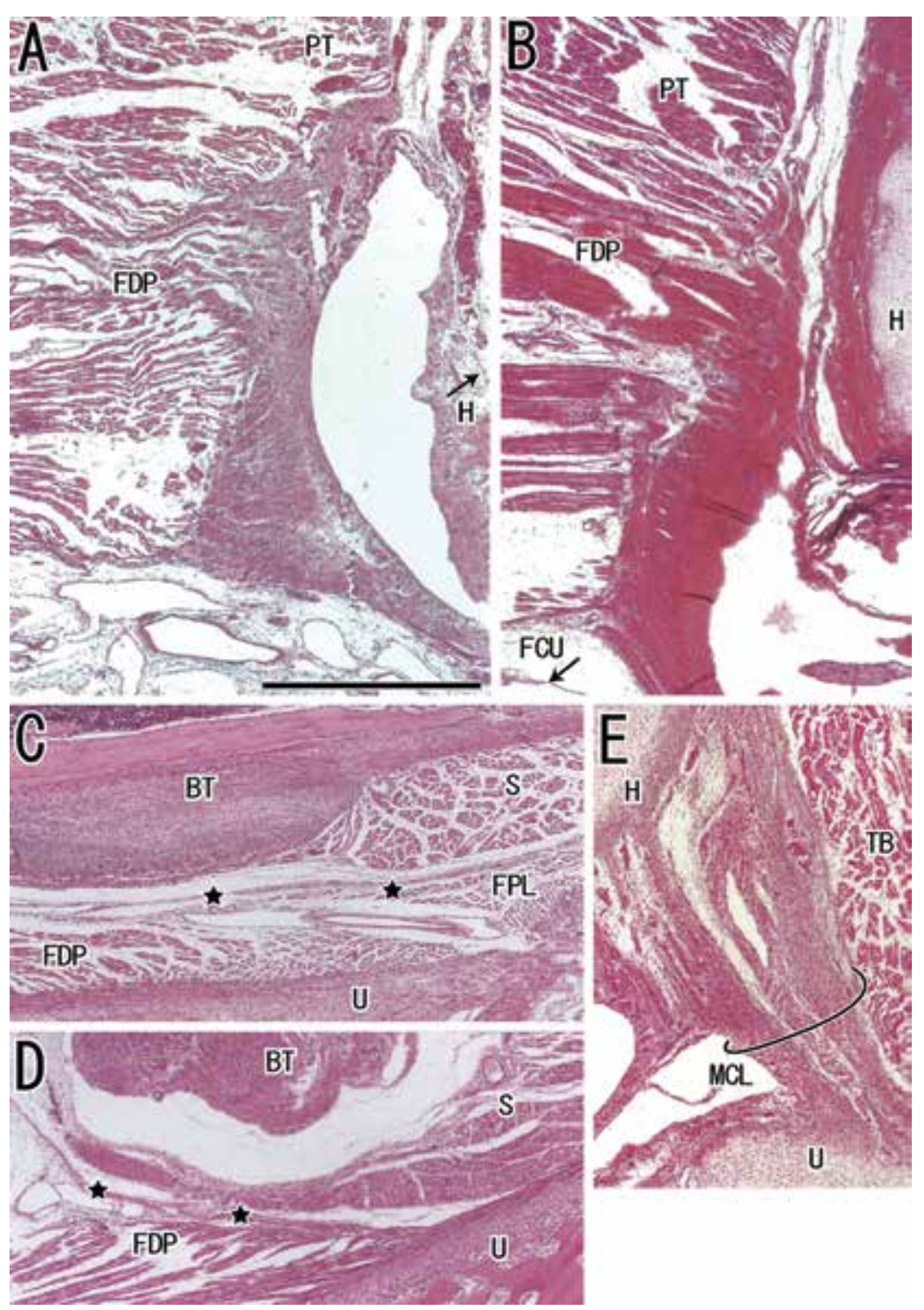

Figure 7. Muscle attachment to the elbow joint capsule. Panels A and $\mathbf{B}$ display the origin of the flexor digitorum profundus muscle (FDP) from the joint capsule: higher magnification views of Figures $5 F$ and $6 F$, respectively. Panels $\mathbf{C}$ and $\mathbf{D}$ exhibit a border area between the supinator (S) and FDP muscles: higher magnification views of Figures $5 \mathrm{~B}$ and $6 \mathrm{C}$, respectively. A fascia (stars) or a candidate of the developing oblique cord is seen at the border. Panel $\mathbf{E}$ shows the medial collateral ligament (MCL) covered by the triceps brachii muscle (TB): a higher magnification view of Figure 5F. All panels are prepared at the same magnification (scale bar in panel A: $1 \mathrm{~mm}$ ); BT — biceps tendon; FCU — flexor carpi ulnaris muscle; FPL — flexor pollicis longus muscle; $\mathrm{H}$ — humerus; PT — pronator teres muscle; $\mathrm{U}$ — ulna.

the posterior and medial aspects of the joint capsule were widely covered by the triceps muscle. The triceps muscle, with its continuation to the anconeus muscle, covered the lateral half of the olecranon of the ulna (Figs. 5B, C; 6B, C). The anconeus muscle appeared to be inserted into the joint capsule. Similarly, the supinator muscle, especially the part superficial to the course of the radial nerve, was attached to the joint capsule near the annular ligament (Figs. 5B; 6B). Notably, the triceps brachii muscle extended medially near the elbow in place of the brachialis muscle. Thus, the medial collateral ligament was not covered by the flexor-pronator muscles but by the triceps muscle (Figs. 5F, G; 6F; 7E). Similarly, rather than being covered by the anconeus muscle, the lateral collateral ligament was covered by the extensor carpi ulnaris muscle (Fig. 6A) and by the extensor carpi radialis longus and brevis muscles (Fig. 5A).

\section{Macroscopic observations}

The present dissection provided the histological observation that the muscle belly of the FDP extended 


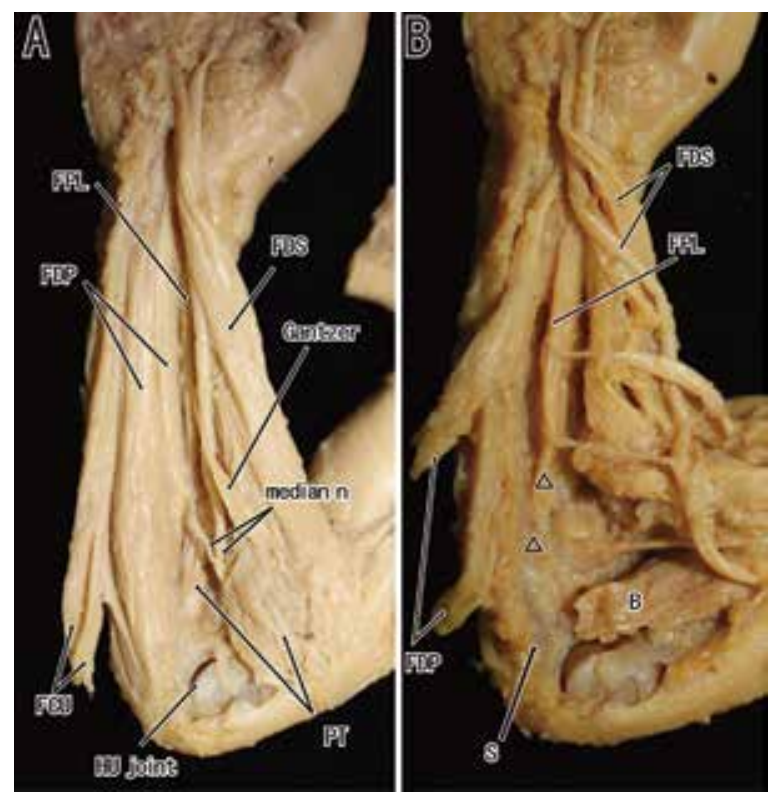

Figure 8. Macroscopic observation of the flexor digitorum profundus muscle at 30 weeks. The flexor digitorum profundus (FDP) muscle extends proximally to reach the elbow joint capsule (A). This specimen carries an additional head (Gantzer) of the flexor pollicis longus muscle (FPL). After removal of the FDP muscle (B), a definite oblique cord is absent but a loose tissue (triangles) is seen extending toward the humeroulnar joint ( $\mathrm{HU}$ joint); $\mathrm{B}$ - brachialis muscle; FCU — flexor carpi ulnaris muscle; FDS — flexor digitorum superficialis muscle; $\mathrm{PT}$ — pronator teres muscle; $\mathrm{S}$ supinator muscle.

proximally to reach the elbow joint (Fig. 8A). Although only 3 specimens were evaluated, 2 showed the additional head (Gantzer's head) of the FPL. Following removal of the FDP, a distinct ligamentous structure suggestive of the oblique cord was not observed; rather, loose fibrous tissue was found to extend toward the humeroulnar joint (Fig. 8B). The FDP was separated from the supinator muscle by the loose tissue.

\section{DISCUSSION}

Although the sections examined in this study included a border area between the supinator muscle and the FPL and FDP muscles, there was no oblique cord-like structure. Rather, a fascia was observed, suggesting that the oblique cord did not form prenatally. However, we found that the FDP extended proximally, originating from the elbow joint capsule. The FPL origin also extended to a site near the capsule. These muscle morphologies differed from those in adults. The proximal parts of the FDP and FPL in foetuses were likely later replaced by collagenous tissues, resulting in a specific type of intermuscular septum i.e., the oblique cord. A muscle-ligament transition from the supinator to the annular ligament of the radius likely occurred very near the cord [10]. In adults, musculofibrous tissues from the supinator muscle belly often join the oblique cord [14]. Likewise, thickening of the foetal subscapularis tendon involves degeneration of parts of the muscle fibres [1]. These findings suggested that the oblique cord formed after birth under the influence of individual mechanical stresses during pronation-supination movements. The postnatal development of the cord (or the regression of the proximal origin of the FDP and FPL muscles) likely occurred in combination with the further thickening and widening of the annular ligament. As it is separated from the FDP [14, 23], the additional head of the FPL was apparently not involved in this hypothetical muscle-ligament transition.

Table 1. Previous reports on the muscle origin from and insertion to the joint capsule in human adults

\begin{tabular}{lll}
\hline Joint name & Muscle origin from or insertion to the capsule & Authors \\
\hline Temporomandibular & Pterygoideus lateralis & Myers, 1988 [17]; Antonopoulou et al., 2013 [4] \\
Glenohumeral & Infraspinatus, teres minor & Clark et al., 1990 [6]; Nimura et al., 2012 [20] \\
& Triceps long head & Handling et al., 2010 [9] \\
& Pectorales major et minor & Tubbs et al., 2005 [29]; 2008b [31] \\
Hip & lliacus, gluteus minimus & Walters et al., 2001 [34], 2014 [33] \\
Knee & Articularis genu & Woodley et al., 2012 [37] \\
& Popliteus & Feipel et al., 2003 [8]; LaPrade et al., 2007 [13] \\
Zygapophysial & Multifidus & Winkelstein et al., 2001 [36] \\
\hline Elbow & Humeroulnar: anconeus & Tubbs et al., 2006 (adult) [30], Present study (foetus) \\
& Flexor digitorum profundus, brachialis & Present study (foetus) \\
& Humeroradial: extensor carpi radialis brevis & Nimura et al., 2014 (adult) [19] \\
& Supinator & Present study (foetus) \\
\hline
\end{tabular}


Along with the wide insertion of the brachialis muscle into the joint capsule, the FDP origin may play a role in avoiding impingement of the foetal joint capsule, a role played in adults by the anconeus [30] and extensor carpi radialis brevis [19] muscles. In foetuses, some fibres of the anconeus muscle were attached to the capsule, whereas those of the extensor carpi radialis brevis muscle did not. To our knowledge, muscles originating from or inserted into adult joint capsules were previously reported in five joints other than the elbow (Table 1), whereas information about foetal joints is very limited. During foetal development, however, capsule impingement was likely to occur due to unbalanced growth between the muscle and bone. The distal, ulnar part of the joint capsule was thickened by the origin of the FDP muscle, whereas the proximal, anterior part of the capsule was retracted by insertion of the brachialis muscle. The FDP origin from the capsule was not reported in adults, whereas the insertion of the brachialis muscle into the capsule had been suspected but not observed in adults [26, 32].

\section{CONCLUSIONS}

The adult elbow joint provides strong muscle support for stabilisation (see the Introduction). However, foetal muscles contributing to elbow support differed from those in adults. The supinator and anconeus muscles were apparently important in both foetuses and adults, whereas the FDP in adults does not reach the joint. The roles of the triceps brachii and brachialis muscles were much greater in foetuses than in adults. Similarly, muscles covering the collateral ligaments differed in foetuses and adults. Therefore, the foetal elbow joint is apparently supported by muscles in a foetus-specific manner. As seen in primitive glenohumeral joints, in which simple collateral ligaments are evident rather than rotator cuff tendons [2], the foetal elbow is not a miniature version of the adult elbow. The topographical relationships between joints and muscles likely change drastically depending on foetal stage.

\section{Acknowledgements}

This work was supported by the National Natural Science Foundation of China (81460471).

\section{REFERENCES}

1. Abe S, Aoki M, Nakao T, Kasahara M, Rodriguez-Vazquez JF, Murakami G, Cho BH (2014) Variation of the subscapularis tendon in and around the fetal glenohumeral joint. Okajimas Folia Anat Jpn, 90: 89-95.
2. Abe S, Nakamura T, Rodríguez-Vázquez JF, Murakami G, IdeY (2011) Early fetal development of the rotator interval region of the shoulder with special reference to topographical relations among related tendons and ligaments. Surg Radiol Anat, 33: 609-615.

3. AbeS, Rhee SK, Osonoi M, Nakamura T, Cho BH, Murakami G, Ide $Y$ (2010) Expression of intermediate filaments at muscle insertions of human fetuses. J Anat, 217: 167-173.

4. Antonopoulou M, latrou I, Paraschos A, Anagnostopoulou S (2013) Variations of the attachment of the superior head of human lateral pterygoid muscle. J Craniomaxillofac Surg, 41: e91-e97.

5. Christensen JB, Adams JP, Cho KO, Miller L (1968) A study of the interosseous distance between the radius and ulna during rotation of the forearm. Anat Rec, 160: 261-272.

6. Clark J, Sidles JA, Matsen FA (1990) The relationship of the glenohumeral joint capsule to the rotator cuff. Clin Orthop Relat Res, 254: 29-34.

7. Davidson PA, Pink M, Perry J, Jobe FW (1995) Functional anatomy of the flexor pronator muscle group in relation to the medial collateral ligament of the elbow. Am J Sport Med, 23: 245-250.

8. Feipel V, Simonnet ML, Rooze M (2003) The proximal attachments of the popliteus muscle: a quantitative study and clinical significance. Surg Radiol Anat, 25: 58-63.

9. Handling MA, Curtis AS, Miller SL (2010) The origin of the long head of the triceps: a cadaveric study. J Shoulder Elbow Surg, 19: 69-72.

10. Hayashi S, Kim JH, Nakano T, Rodríguez-Vázquez JF, Murakami G, Fukuzawa Y (2013) Influence of developing ligaments on the muscles in contact with them: a study of the annular ligament of the radius and the sacrospinous ligament in mid-term human fetuses. Anat Cell Biol, 46: 149-156.

11. Hayner JC (1945). Muscular associations of the oblique cord. Anat Rec, 91: 280.

12. Kim PT, Isogai S, Murakami G, Wada T, Aoki M, Yamashita T, Ishii S (2002) The lateral collateral ligament complex and related muscles act as a dynamic stabilizer as well as a static supporting structure at the elbow joint: an anatomical and experimental study. Okajimas Folia Anat Jpn, 79: 55-62.

13. LaPrade RF, Morgan PM, Wentorf FA, Johansen S, Engebretsen $L$ (2007) The anatomy of the posterior aspect of the knee. An anatomic study. J Bone Joint Surg Am, 89: 758-764.

14. Martin BF (1958) The oblique cord of the forearm. J Anat, 95: 609-615.

15. McGinley JC, D'Addressi L, Sadeghipour K, Kozin SH (2001) Mechanics of the antebrachial interosseous membrane: response to sharing forces. J Hand Surg Am, 26: 733-741.

16. Morrey BF, An KN (1985) Functional anatomy of the ligaments the elbow. Clin Orthop, 201: 84-90.

17. Myers $\amalg$ (1988) Newly described muscle attachments to the anterior band of the articular disk of the temporomandibular joint. J Am Dent Assoc, 117: 437-439.

18. Nakamura T, Yabe $Y$, Horiuchi $Y$ (1999) In vivo MR studies of dynamic changes in the interosseous membrane of the forearm during rotation. J Hand Surg, 24: 245-248.

19. Nimura A, Fujishiro H, Wakabayashi $Y$, Imatani J, Sugaya $H$, Akita K (2014) Joint capsule attachment to the exten- 
sor carpi radialis brevis origin: an natomical study with possible implications regarding the etiology of lateral epicondylitis. J Hand Surg Am, 39: 219-225.

20. Nimura A, Kato A, Yamaguchi K, Mochizuki T, Okawa A, Sugaya H, Akita K (2012) The superior capsule of the shoulder joint complements the insertion of the rotator cuff. J Shoulder Elbow Surg, 21: 867-842.

21. O'Driscoll SW, Horii E, Morrey BF, Carmichael SW (1992) Anatomy of the ulnar part of the lateral collateral ligament of the elbow. Clin Anat, 5: 296-303.

22. Otoshi K, Kikuchi S, Shishido H, Konno S (2014) The proximal origins of the flexor-pronator muscles and their role in the dynamic stabilization of the elbow: an anatomical study. Surg Radiol Anat, 36: 289-294.

23. Pai MM, Nayak SR, Krishnamurthy A, Vadgaonkar R, Prabhy LV, ranada AV, Janardhan JP, Rai $R$ (2008) The accessory heads of flexor pollicis longus and flexor digitorum profundus: incidence and morphology. Clin Anat, 21: 252-258.

24. Patel BA (2005) Form and function of the oblique cord (corda obliqua) in anthropoid primates. Primates, 46: 47-57.

25. Pfaeffle HJ, Fischer KJ, Manson TT, Tomaino MM, Herndon $J H$, Woo SLY (1999) A new methodology to measure load transfer through the forearm using multiple force sensors. J Biomech, 32: 1331-1335.

26. Sanal HT, Chen L, Negrao P, Haghighi P, Trudell DJ, Resnick DL (2009) Distal attachment of the brachialis muscle: anatomic and MRI study in cadavers. AJR, 192: 468-472.

27. Shepard MF, Markolf KL, Dunbar AM (2001) The effects of partial and total interosseous membrane transection on load sharing in the cadaver forearm. J Orthop Res, 19: 587-592.

28. Skahen JR, Palmer AK, Werner FW, Fortino MD (1997) The interosseous membrane of the forearm: anatomy and function. J Hand Surg, 22: 981-985.

29. Tubbs RS, Oakes WJ, Salter EG (2005) Unusual attachment of the pectoralis minor muscle. Clin Anat, 18: 302-304.

30. Tubbs RS, Oakes WJ, Salter EG (2006) The subanconeus muscle. Folia Morphol, 65: 22-25.

31. Tubbs RS, Shoja MM, Shokouhi G, Loukas M, Oakes WJ (2008b) Insertion of the pectoralis major into the shoulder joint capsule. Anat Sci Int, 83: 291-293.

32. Tubbs RS, Yablick NW, Loukas M, Shoja MM, Ardalan M, Oakes WJ (2008a) Capsular attachment of the brachialis muscle (Portal's muscle): an anatomicval and functional study. Surg Radiol Anat, 30: 229-232.

33. Walters BL, Cooper JH, Rodriguez JA (2014) New findings in hip capsular anatomy: dimensions of capsular thickness and pericapsular contributions. Arthroscopy 30: 1235-1245.

34. Walters J, Solomons M, Davies J (2001) Gluteus minimus: observations on its insertion. J Anat, 198: 239-242.

35. Williams PL (1995) Gray's Anatomy. 38th Ed. Churchill Livingstone, Edinburgh.

36. Winkelstein BA, McLendon RE, Barbir A, Myers BS (2001) An anatomical investigation of the human cervical facet capsule, quantifying muscle insertion area. J Anat, 198: 455-461.

37. Woodley SJ, Latimer CP, Meikle GR, Stringer MD (2012) Articularis genu: an anatomic and MRI study in cadavers. J Bone Joint Surg Am, 94: 59-67. 